\title{
Erratum: Nonlinearities and effects of transverse beam size in beam position monitors [Phys. Rev. ST Accel. Beams 4, 092801 (2001)]
}

\author{
Sergey S. Kurennoy \\ (Received 12 February 2003; published 13 March 2003)
}

DOI: 10.1103/PhysRevSTAB.6.039902

PACS numbers: 41.75.-i, 41.20.-q, 99.10.Cd

In the original paper, some 5th-order terms in Eqs. (7), (22), and (25) for the difference-over-sum ratio of the induced signals on the beam position monitor (BPM) electrodes were incorrect. These errors led to incorrect Figs. 2-9. The corrected equations and corresponding figures are presented below. For convenience, we keep the same numbering of equations and figures as in the original text. The complete revised text is available electronically [1].

In a circular chamber of radius $b$ with a pair of strip line electrodes in the horizontal plane, which have subtended angle $\phi$ per strip line, the ratio of the difference between the signals on the right $(R)$ and left $(L)$ electrodes to the sum of these signals is

$$
\begin{aligned}
\frac{R-L}{R+L}=2 \frac{x_{0}}{b} \frac{\sin (\phi / 2)}{\phi / 2}\{ & 1-\frac{2}{b^{2}} \frac{\sin \phi}{\phi}\left(\sigma_{x}^{2}-\sigma_{y}^{2}+x_{0}^{2}-y_{0}^{2}\right)+\frac{1}{b^{2}} \frac{\sin (3 \phi / 2)}{\sin (\phi / 2)}\left(\sigma_{x}^{2}-\sigma_{y}^{2}+x_{0}^{2} / 3-y_{0}^{2}\right) \\
& -\frac{2}{b^{4}} \frac{\sin \phi}{\phi} \frac{\sin (3 \phi / 2)}{\sin (\phi / 2)}\left(\sigma_{x}^{2}-\sigma_{y}^{2}+x_{0}^{2}-y_{0}^{2}\right)\left(\sigma_{x}^{2}-\sigma_{y}^{2}+x_{0}^{2} / 3-y_{0}^{2}\right) \\
& -\frac{2}{b^{4}} \frac{\sin (2 \phi)}{2 \phi}\left[3\left(\sigma_{x}^{2}-\sigma_{y}^{2}+x_{0}^{2}-y_{0}^{2}\right)^{2}-2 x_{0}^{4}-2 y_{0}^{4}\right]+\frac{4}{b^{4}}\left(\frac{\sin \phi}{\phi}\right)^{2}\left(\sigma_{x}^{2}-\sigma_{y}^{2}+x_{0}^{2}-y_{0}^{2}\right)^{2} \\
& \left.+\frac{1}{b^{4}} \frac{\sin (5 \phi / 2)}{\sin (\phi / 2)}\left[3\left(\sigma_{x}^{2}-\sigma_{y}^{2}\right)^{2}+2\left(\sigma_{x}^{2}-\sigma_{y}^{2}\right)\left(x_{0}^{2}-3 y_{0}^{2}\right)+x_{0}^{4} / 5-2 x_{0}^{2} y_{0}^{2}+y_{0}^{4}\right]+O\left(b^{-6}\right)\right\} .
\end{aligned}
$$

Corrections (7) are shown in Figs. 2-5 for a $60^{\circ}$ strip line BPM. Figure 2 shows the nonlinearities of the BPM response for a pencil beam. The changes of this signal ratio when the beams become flat (Figs. 3 and 4 ) are practically unnoticeable. In Fig. 5 the ratio $S / S_{0}$, where $S=(R-L) /(R+L)$ for a finite-size beam, and $S_{0}$ is the same ratio for a pencil beam, is plotted versus the beam center position. One can conclude from Fig. 5 that in this BPM for a reasonable transverse beam size the beam-size corrections are on the level of $1 \%$. A weak dependence of the signal ratio on the beam size and on the vertical beam offset $y_{0}$ in this case is mainly due to the fact that the BPM electrodes are wide. For narrow electrodes the beam-size and transverse coupling effects are stronger, on the order of a few percent; see Figs. 6-9.

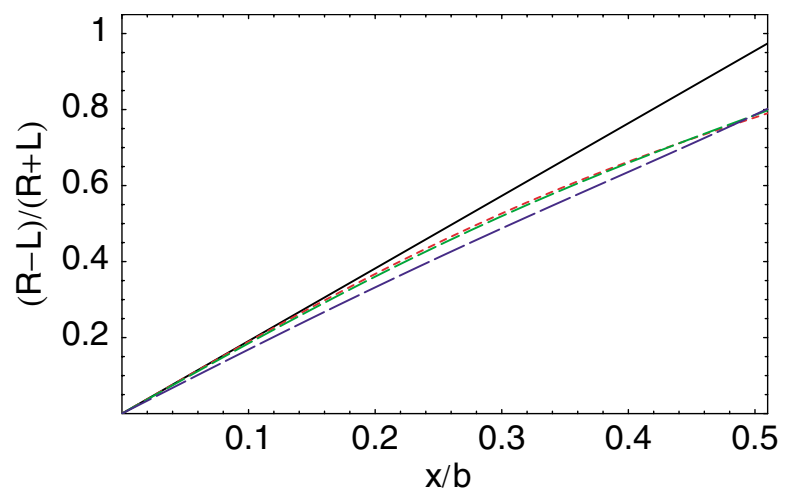

FIG. 2. (Color) BPM signal ratio (7) in a circular chamber versus beam center position $x / b$ for three vertical beam offsets $y / b=0,1 / 4,1 / 2$ (short-dashed, dashed, long-dashed lines) without beam-size corrections (pencil beam, $\sigma_{x}=\sigma_{y}=0$ ). Solid line shows the linear part of the BPM response. 


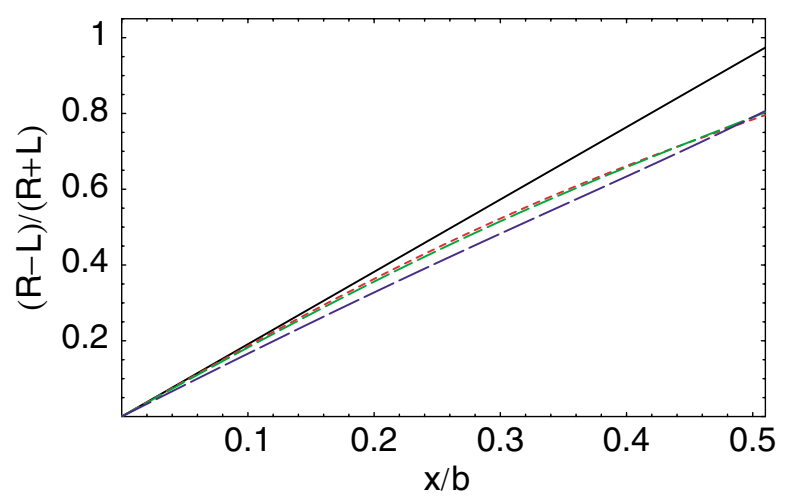

FIG. 4. (Color) Same as Fig. 3, but with $\sigma_{x}=0, \sigma_{y} / b=0.2$.

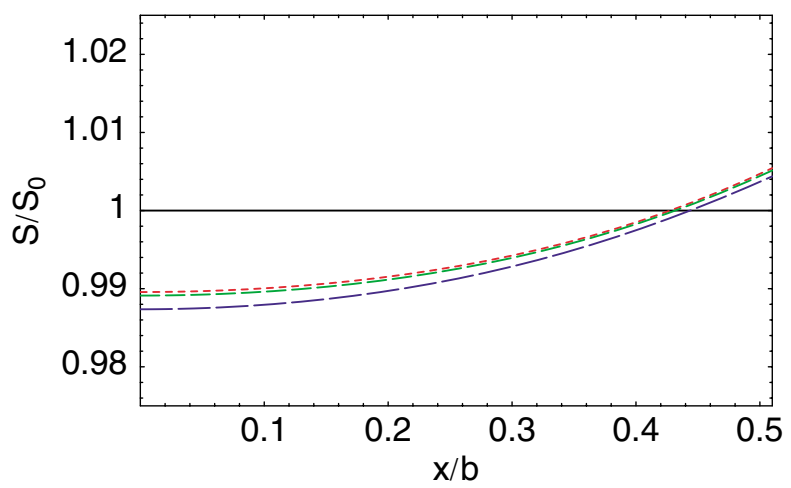

FIG. 5. (Color) Relative magnitude of beam-size corrections in a circular chamber with $\sigma_{x} / b=0.1, \sigma_{y} / b=0.2$ for three vertical beam offsets $y / b=0,1 / 4,1 / 2$ (short-dashed, dashed, long-dashed lines). Here 1 corresponds to a pencil beam case, i.e., to one of the three curves in Fig. 2 for the corresponding beam vertical offset.

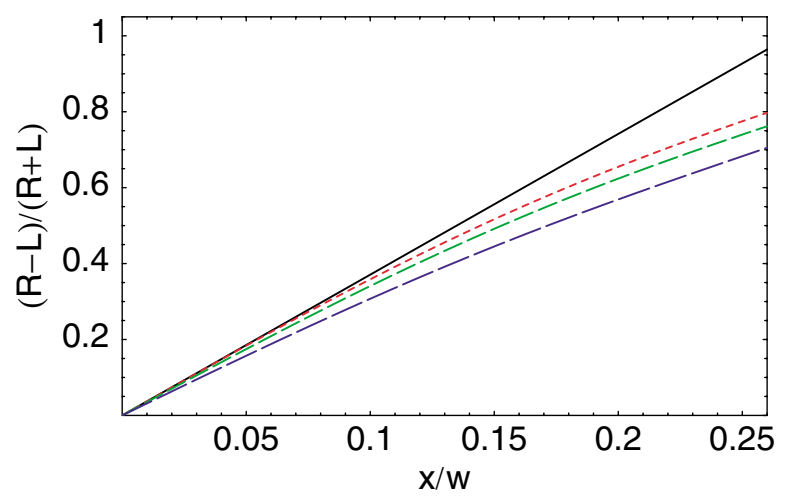

FIG. 6. (Color) BPM signal ratio (25) in a square chamber versus beam center position $x / w$ for three vertical beam offsets $y / h=0,1 / 8,1 / 4$ (short-dashed, dashed, long-dashed lines) without beam-size corrections (pencil beam, $\sigma_{x}=\sigma_{y}=0$ ). Solid line shows the linear part of the BPM response.

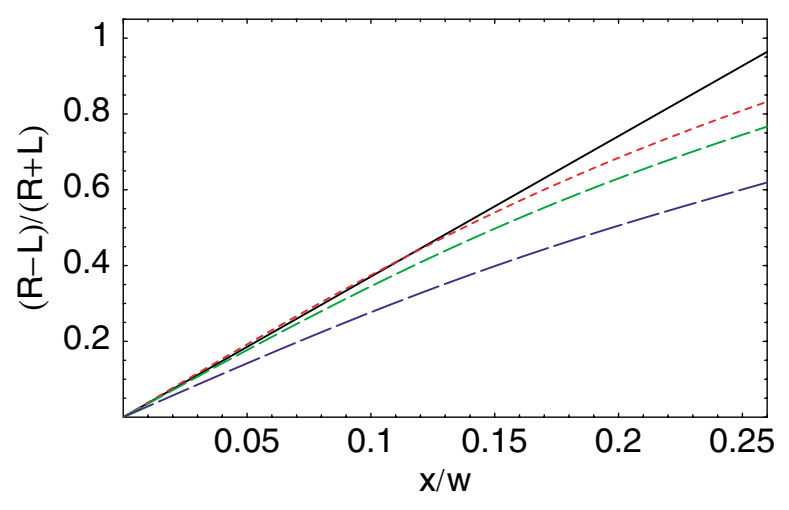

FIG. 7. (Color) Same as Fig. 6, but with beam-size corrections for a flat beam with $\sigma_{x} / w=0.1, \sigma_{y}=0$.

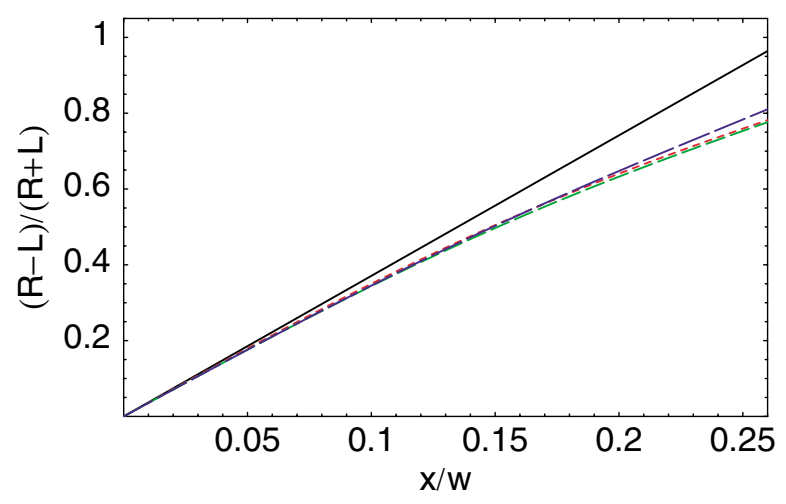

FIG. 8. (Color) Same as Fig. 7, but with $\sigma_{x}=0, \sigma_{y} / h=0.1$.

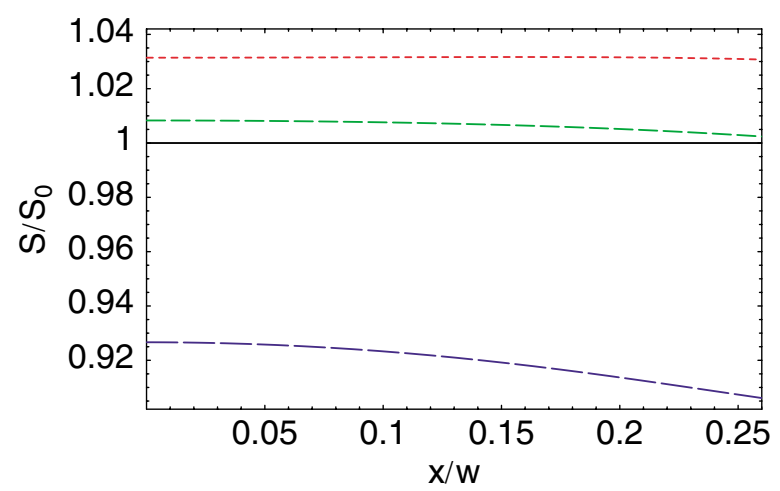

FIG. 9. (Color) Relative magnitude of beam-size corrections in a square chamber with $\sigma_{x} / w=0.1, \sigma_{y} / h=0.05$ for three vertical beam offsets $y / h=0,1 / 8,1 / 4$ (short-dashed, dashed, long-dashed lines). Here 1 corresponds to a pencil beam case, i.e., to one of the three curves in Fig. 6 for the corresponding beam vertical offset. 
For the case of a BPM cross section that is arbitrary but symmetric with respect to its vertical and horizontal axes, and a horizontal pair of the electrodes, the difference-over-sum signal ratio of BPM signals is

$$
\begin{aligned}
\frac{R-L}{R+L}=\frac{x_{0} \partial_{x} e_{0}}{e_{0}}\{ & 1+\frac{1}{2} \frac{\partial_{x}^{3} e_{0}}{\partial_{x} e_{0}}\left(\frac{x_{0}^{2}}{3}-y_{0}^{2}+M_{2}\right)-\frac{1}{2} \frac{\partial_{x}^{2} e_{0}}{e_{0}}\left(x_{0}^{2}-y_{0}^{2}+M_{2}\right)+\frac{1}{4}\left(\frac{\partial_{x}^{2} e_{0}}{e_{0}}\right)^{2}\left(x_{0}^{2}-y_{0}^{2}+M_{2}\right)^{2} \\
& -\frac{1}{4} \frac{\partial_{x}^{3} e_{0}}{\partial_{x} e_{0}} \frac{\partial_{x}^{2} e_{0}}{e_{0}}\left(\frac{x_{0}^{2}}{3}-y_{0}^{2}+M_{2}\right)\left(x_{0}^{2}-y_{0}^{2}+M_{2}\right)+\frac{1}{24} \frac{\partial_{x}^{5} e_{0}}{\partial_{x} e_{0}}\left[\frac{x_{0}^{4}}{5}-2 x_{0}^{2} y_{0}^{2}+y_{0}^{4}+2 M_{2}\left(x_{0}^{2}-3 y_{0}^{2}\right)+M_{4}\right] \\
& \left.-\frac{1}{24} \frac{\partial_{x}^{4} e_{0}}{e_{0}}\left[x_{0}^{4}-6 x_{0}^{2} y_{0}^{2}+y_{0}^{4}+6 M_{2}\left(x_{0}^{2}-y_{0}^{2}\right)+M_{4}\right]+O\left(r_{0}^{6} / b^{6}, \sigma^{6} / b^{6}\right)\right\},
\end{aligned}
$$

where we implicitly assume values of the field $e_{0}$ and its derivatives averaged over the transverse extent of the right $(R)$ electrode. It means that for brevity $\partial_{x}^{n} e_{0}, n=0,1,2, \ldots$, stands here for $\overline{\partial_{x}^{n} e_{0}}=1 / \tau_{R} \int_{R} \partial_{x}^{n} e_{0}(\tau) d \tau$, where $\tau$ is a tangential length parameter of the electrode in its transverse cross section, and $\tau_{R}=\int_{R} d \tau$ is the electrode transverse width.

It takes a small effort to arrive from the general expression (22) to its particular case for the circular pipe, Eq. (7). We just note that for the circular pipe $\overline{e_{0}}=e_{0}=1 /(2 \pi b)$ and

$$
\partial_{x}^{n} e_{0}=\frac{n ! \cos n \theta}{\pi b^{n+1}}, \quad n=1,2, \ldots
$$

Averaging over the right electrode azimuthal extent, $-\phi / 2 \leq \theta \leq \phi / 2$, one gets

$$
\overline{\partial_{x}^{n} e_{0}}=2 \frac{(n-1) ! \sin (n \phi / 2)}{\pi b^{n+1}}, \quad n=1,2, \ldots
$$

After that obtaining Eq. (7) from (22) is straightforward, taking into account the expressions for the distribution moments $M_{2}, M_{4}$ of a double-Gaussian beam.

For two strip line BPM electrodes of width $h_{1}$ on sidewalls of a rectangular vacuum chamber $w \times h$, the differenceover-sum signal ratio, up to the 5 th order, is

$$
\begin{aligned}
\frac{R-L}{R+L}=\pi \frac{x_{0}}{h} \frac{\Sigma_{1}}{\Sigma_{0}}\{ & 1+\frac{\pi^{2}}{2 h^{2}} \frac{\Sigma_{3}}{\Sigma_{1}}\left(\frac{x_{0}^{2}}{3}-y_{0}^{2}+M_{2}\right)-\frac{\pi^{2}}{2 h^{2}} \frac{\Sigma_{2}}{\Sigma_{0}}\left(x_{0}^{2}-y_{0}^{2}+M_{2}\right)+\frac{\pi^{4}}{4 h^{4}} \frac{\Sigma_{2}^{2}}{\Sigma_{0}^{2}}\left(x_{0}^{2}-y_{0}^{2}+M_{2}\right)^{2} \\
& -\frac{\pi^{4}}{4 h^{4}} \frac{\Sigma_{3}}{\Sigma_{1}} \frac{\Sigma_{2}}{\Sigma_{0}}\left(\frac{x_{0}^{2}}{3}-y_{0}^{2}+M_{2}\right)\left(x_{0}^{2}-y_{0}^{2}+M_{2}\right)+\frac{\pi^{4}}{24 h^{4}} \frac{\Sigma_{5}}{\Sigma_{1}}\left[\frac{x_{0}^{4}}{5}-2 x_{0}^{2} y_{0}^{2}+y_{0}^{4}+2 M_{2}\left(x_{0}^{2}-3 y_{0}^{2}\right)+M_{4}\right] \\
& \left.-\frac{\pi^{4}}{24 h^{4}} \frac{\Sigma_{4}}{\Sigma_{0}}\left[x_{0}^{4}-6 x_{0}^{2} y_{0}^{2}+y_{0}^{4}+6 M_{2}\left(x_{0}^{2}-y_{0}^{2}\right)+M_{4}\right]+O\left(r_{0}^{6} / b^{6}, \sigma^{6} / b^{6}\right)\right\}
\end{aligned}
$$

where sums $\Sigma_{n}$ are defined in the original text.

Corrections (25) are shown in Figs. 6-9 for a square chamber, $w=h$, and a BPM with very narrow electrodes, $h_{1}=h / 100$ (in fact, results for $h_{1}=h / 10$ are almost identical). Figure 6 shows the BPM nonlinearities for a pencil beam. Comparing it with the signal ratios for flat beams in Figs. 7 and 8, we notice a significant dependence of the signal ratio on the beam shape. Similar to Fig. 5, in Fig. $9 S=(R-L) /(R+L)$ for a finite-size beam, while $S_{0}$ is the same ratio for a pencil beam, which is plotted in Fig. 6. Therefore, $S / S_{0}=1$ in Fig. 9 would mean that there was no correction due to a finite transverse beam size. As one can see, the beam-size corrections here are rather large, and they also depend noticeably on the beam vertical offset. The corrections range from about $+3 \%$ for $y=0$ (the chamber midplane) to less than $1 \%$ for $y=h / 8$ to about $-(7-10) \%$ for $y=h / 4$ (the beam is halfway to the top wall), in the case of $\sigma_{x} / w=0.1$, $\sigma_{y} / h=0.05$ shown in Fig. 9 .

These results are quite different from those for the wide-electrode BPM in a circular chamber, where the beam-size corrections were rather small. This is mainly because the considered square BPM has narrow electrodes and not due to the different shape of its cross section.

[1] S. S. Kurennoy, physics/0108015. 